\title{
A Short Review of Textile Applications in Antenna Design
}

\author{
Bin Xu*1, Rachel Eike ${ }^{2}$, Allyson Cliett ${ }^{2}$, Rinn Cloud ${ }^{2}$ and Yang Li ${ }^{1}$ \\ ${ }^{1}$ Electrical and Computer Engineering, Baylor University, USA \\ ${ }^{2}$ Family and Consumer Sciences, Baylor University, USA \\ *Corresponding author: Bin Xu, Baylor University, One Bear Place \#97356 Waco, TX 76798, USA
}

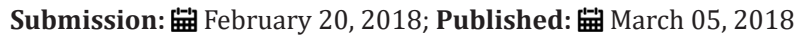

\begin{abstract}
This review briefly introduces the motivation of applying e-textiles to antenna design and some examples of textile antenna. The objective is to help textile engineers gain insight of desired e-textile properties for antenna design and provide assistance in the development of new textiles to improve antenna performance.

Keywords: E-textile; Textile Antenna; Surface resistivity; Permittivity; Smart clothing

Abbreviations: DC: Direct Current; E-Textiles: Electronic Textiles; RF: Radio Frequency; MF: Microwave Frequency; WBAN: Wireless Body Area Networks
\end{abstract}

\section{Introduction}

The market for wearable technology has been slowly growing since the 1970s and has dramatically increased in recent years from the popularity of fitness trackers and smart watches. Many wearables are used for collecting health related data and communicating data with devices. It may be convenient to integrate wearables directly into clothing (also called smart clothing or e-clothing) instead of attaching them onto the body or garment. However, wearable technologies have not been fully integrated into clothing because traditional antenna or circuits are made of rigid materials, which make clothing integration challenging. E-textile antennas and circuits, which are made of conductive fabrics, show great potential to implement wearables into garments due to their low mass, physical flexibility and ability to be sewn for garment construction.

\section{Development of textile in electronics}

The application of textiles in electrical engineering (i.e., e-textiles or smart clothing) can date back to 1993. Wong explored the possibility of using conductive polymer composites as radar absorbers [1]. A few years later, Wong studied a new smart material, which was based on this conducting polymer composite material, and measured DC and MF electrical behaviors [2]. Rapid development of the semiconductor industry and communication technologies led to computer systems becoming much more light weight and compact than before. Compaction of electronic components has given option for clothing embedding making smart clothing a practical garment to use in everyday life. Both conducting and non-conducting textiles are finding more and more applications in smart clothing design.

\section{Discussion}

\section{Electrical properties of textiles}

To understand the effect of applying textile materials into electrical circuit, it is important to know their electrical properties for both conducting and non-conducting textiles.

Table 1: Surface resistivity of some commercial e-textiles [3].

\begin{tabular}{|c|c|}
\hline E-Textile & Surface Resistivity (Ohm/Square) \\
\hline Silver jersey knit fabric & 2 \\
\hline Silverell fabric & 5 \\
\hline Nickel/Copper ripstop fabric & 0.03 \\
\hline $\begin{array}{c}\text { Pure copper polyester taffeta } \\
\text { fabric }\end{array}$ & 0.05 \\
\hline Ripstop silver fabric & 0.25 \\
\hline
\end{tabular}

Conducting textiles: There are two common approaches to make conducting textiles. One approach is to make e-textile by using metal coated conductive threads. The other approach is to coat a metal layer on top of a normal fabric. For conducting textiles; one of the most important electrical parameters is surface resistivity, which represents the effectiveness of textiles to carry electrical currents. Surface resistivity can be measured following AATCC method 76 [3]. It is also a good indication of their conductivity at 
$\mathrm{RF}$ or MF and determines the antenna efficiency. Table 1 lists the surface resistivity of some e-textiles readily available on market [4]. It is found that knitted e-textiles usually have a higher surface resistivity than woven e-textiles (Table 1 ).

Table 2: Permittivity of commonly used fabrics [4].

\begin{tabular}{|c|c|}
\hline Fabrics & Permittivity \\
\hline Wash cotton & 1.51 \\
\hline Curtain cotton & 1.47 \\
\hline Polyester & 1.44 \\
\hline Polycot & 1.56 \\
\hline Jeans cotton & 1.67 \\
\hline Floor spread & 1.46 \\
\hline
\end{tabular}

Non-conducting textiles: Non-conducting textiles, such as cotton, denim, and polycot, play an important role in smart clothing, particularly in antenna design. These textiles may be used as a substrate, which is the supporting structure for antennas. Thus, its permittivity is a key parameter to consider for textile antenna design as it may affect the antenna size and bandwidth. Different methods have been conducted to measure the permittivity of textiles. Sankaraling proposed calculating resonance frequency of a patch antenna to measure permittivity [5]. Table 2 lists the measured permittivity of commonly used substrate fabrics.

\section{Textile applications in antenna}

In general, wearable antenna designs need to be low profile, light weight, small volume, and low production cost. E-textile antennas show great potential for wearable antenna design due to their low mass, physical flexibility and ease of integration with garments [6]. Wearable antennas require the e-textiles to have a low surface resistivity. E-textiles with surface resistivity below $0.050 \mathrm{hm} /$ Square can achieve comparable electrical performance with traditional copper antennas. Many textile antennas have been designed to work at different frequency bands for different applications and on different locations on the human body. Figure 1 shows three examples of textile antennas [7-9].They are designed to work at different frequency bands for different applications, such as cellular communication, digital television, WFI, Bluetooth etc. Besides antenna design, some researchers have studied the environmental changes or wearing conditions effect on textile antenna performance, such as shape distortion and moisture effect. For example, Annelien [10] found that increasing the moisture content of a textile antenna could increase the permittivity and loss tangent of the material, therefore, changing antenna resonance frequency and decreasing antenna efficiency.

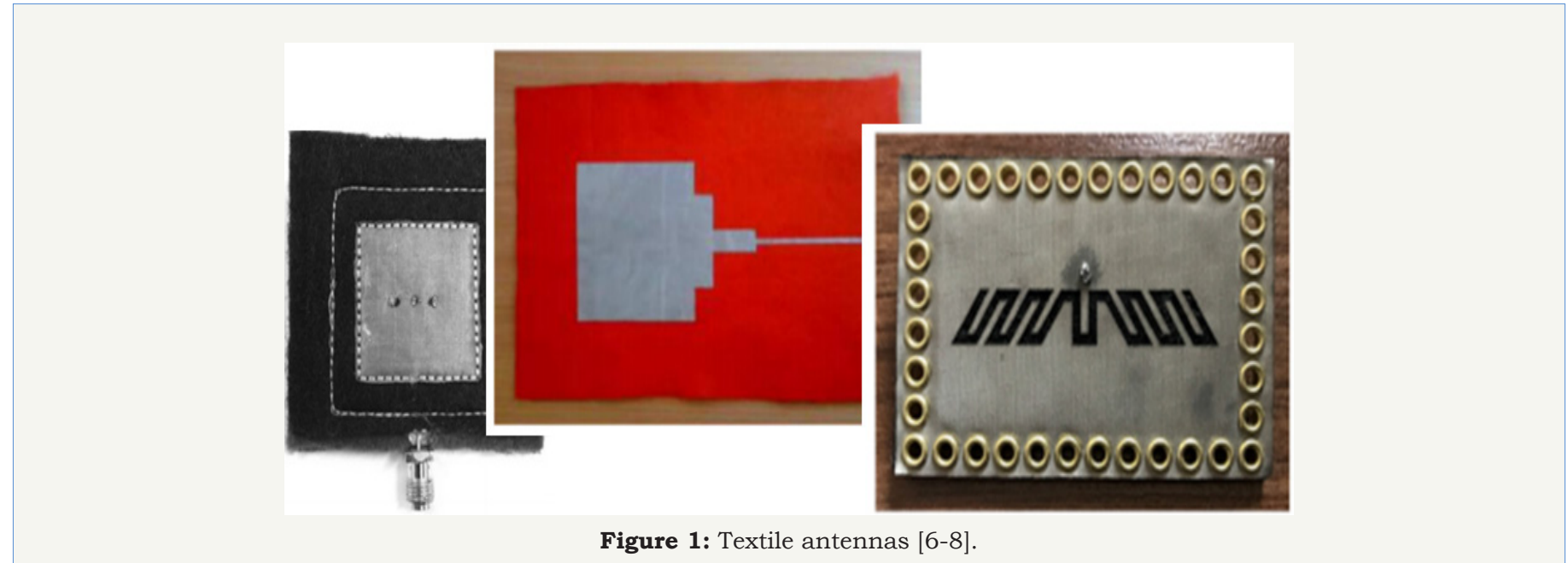

\section{Conclusion}

This review briefly introduced textile applications in antenna design and highlighted some of the developments in e-textiles, including surface resistivity. Non-conducting textiles that serve as substrates for antenna design are explained, followed by three examples of textile antennas. Textile-based antennas showpotentialinwearable devices to communicate a variety of information with other electronics or computers.

\section{References}

1. Wong TP, Chamber B, Anderson A, Wright PV (1993) Fabrication and evaluation of conducting polymer composites as radar absorbers. Eighth International Conference on Antennas and Propagation, Edinburgh, UK.

2. Wong TP, Barnes A, Chambers B, Anderson AP, Wright PV (1997) Microwave characterization of smart materials based on conducting polymer composite material. Tenth International Conference on Antennas and Propagation, Edinburgh, UK.

3. AATCC Standards.

4. Less EMF conductive fabrics.

5. Sangaralingam S, Gupta B (2010) Determination of dielectric constant of fabric materials and their use as substrates for design and development of antennas for wearable applications. IEEE Transactions on Instrumentation and Measurement 59(12): 3122-3130.

6. Hertleer C, Tronquo A, Rogier H, Langenhove LV (2008) The use of textile materials to design wearable microstrip patch antennas. Textile Research Journal 78: 651-658.

7. Paraskevopoulos A, Fonseca D, Seager R, Whittow W, Vardaxoglou J, Alexandridis A (2016) Higher-mode textile patch antenna with embroidered vias for on-body communication. IET Microwave, Antenna \& Propagation 10: 802-807. 
8. Paul D, Giddens H, Paterson MG, Hilton G, Mcgeehan J (2013) Impact of body and clothing on a wearable textile dual band antenna at digital television and wireless communications bands. IEEE Transactions on Antennas and Propagation 61: 2188-2194.

9. Lajevardi M, Kamyab M (2017) Ultra miniaturized meta material inspired SIW textile antenna for off-body applications. IEEE Antennas and Wireless Propagation Letters 16: 3155-3158.

10. Hertleer C, Laere AV, Rogier H, Langenhove LV (2010) Influence of relative humidity on textile antenna. Textile Research Journal 80: 177183.

Your subsequent submission with Crimson Publishers will attain the below benefits

- High-level peer review and editorial services

- Freely accessible online immediately upon publication

- Authors retain the copyright to their work

- Licensing it under a Creative Commons license

- Visibility through different online platforms

- Global attainment for your research

- Article availability in different formats (Pdf, E-pub, Full Text)

- Endless customer service

- Reasonable Membership services

- Reprints availability upon request

- One step article tracking system 